\title{
Correction to: Ecological and Syndemic Predictors of Drug Use During Sex and Transactional Sex among U.S. Black Men Who Have Sex with Men: A Secondary Data Analysis from the HPTN 061 Study
}

\author{
Natalie M. Leblanc ${ }^{1} @ \cdot$ Hugh F. Crean ${ }^{1} \cdot$ Typhanye P. Dyer $^{2} \cdot$ Chen Zhang $^{1} \cdot$ Rodman Turpin $^{2} \cdot$ Nanhua Zhang $^{3}$. \\ Martez D. R. Smith ${ }^{1}$. James McMahon ${ }^{1}$ - LaRon Nelson ${ }^{4}$
}

Published online: 11 May 2021

(c) Springer Science+Business Media, LLC, part of Springer Nature 2021

\section{Correction to: Archives of Sexual Behavior https://doi.org/10.1007/s10508-020-01871-z}

The affiliation given for coauthor Typhanye P. Dyer in this article as originally published was incorrect.

Dr. Dyer's correct affiliation is:

School of Public Health, University of Maryland, College Park, MD, USA.

The original article has been updated.

Publisher's Note Springer Nature remains neutral with regard to jurisdictional claims in published maps and institutional affiliations.

The original article can be found online at https://doi.org/10.1007/ s10508-020-01871-z.

Natalie M. Leblanc

Natalie_leblanc@urmc.rochester.edu

1 School of Nursing, University of Rochester, 601 Elmwood Ave, Box SON, Rochester, NY 14623, USA

2 School of Public Health, University of Maryland, College Park, MD 20742, USA

3 Division of Biostatistics and Epidemiology, Cincinnati Children's Hospital Medical Center, University of Cincinnati College of Medicine, Cincinnati 45267-0056, USA

4 Yale University School of Nursing, New Haven, CT 06477, USA 